\title{
Intraocular Lens Dislocation into the Anterior Chamber because of Repeated Eye-Poking in a Patient with Leber's Congenital Amaurosis
}

\author{
Abdullah A. Al-Owaid ${ }^{a, b}$ Motazz A. Alarfaja, Faris A. Alarfaja, \\ Abdulaziz Awad ${ }^{\mathrm{a}}$ \\ aKing Khaled Eye Specialist Hospital, Riyadh, Saudi Arabia; ${ }^{b}$ Department of \\ Ophthalmology, College of Medicine, Imam Abdulrahman Bin Faisal University, \\ Dammam, Saudi Arabia
}

\section{Keywords}

Leber's congenital amaurosis · Retinal dystrophy · Eye rubbing · Dislocated IOL

\begin{abstract}
The dislocation of a posterior chamber intraocular lens (PC IOL) because of constant eye rubbing is unusual and has never been reported in a child with Leber's congenital amaurosis (LCA) A 4-year-old full-term girl with an ocular history of LCA presented to the emergency room with a single-piece PC IOL dislocated into the anterior chamber (AC) of the left eye. There was no history of trauma or any other surgical intervention. A specific behavior known as Franceschetti's oculo-digital sign is a characteristic feature of LCA; this sign consists of repeated pressing, poking, and rubbing of the eyes with knuckles and fingers to stimulate the photoreceptors. This behavior caused the dislocation of the PC IOL into the AC. The dislocated IOL was explanted, and the patient was provided with aphakic glasses.

(C) 2020 The Author(s) Published by S. Karger AG, Basel
\end{abstract}


 Ophthalmology}

\section{Introduction}

Leber's congenital amaurosis (LCA) was first described as a congenital form of retinitis pigmentosa (RP). LCA is an inherited retinal dystrophy causing blindness by the age of 1 year, with an estimated incidence of 1 in 40,000 newborns [1]. Clinically and genetically, it is a heterogeneous disorder and presents with severe visual impairment since birth. Visual impairment varies from "no light perception" to 20/50, depending on the causative genetic mutation. LCA is usually diagnosed in infancy, and most patients present with searching nystagmus and photophobia [2]. Twenty-two genotypes have been described in the literature based on the causative genetic mutation, and they only account for half of the LCA cases, with CEP290 (15\%), GUCY2D (12\%), CRB1 (10\%), and RPE65 (8\%) being the most common [3].

The retinal phenotype in LCA is widely variable and includes: (1) a relatively normal retina; (2) typical retinal findings of RP, such as vascular attenuation and bone-spicule pigmentation; (3) nummular pigmentation, maculopathy with atrophic changes, or colobomatouslike macular changes; (4) thickening of the macula with sparing of the para-arteriolar retinal pigmental epithelium; and (5) coats-like exudative vasculopathy. Some phenotypic-genotypic correlations are characteristic to some fundus changes [4].

Diagnostic findings such as non-recordable severely diminished rod and cone responses on electroretinography and specific optical coherence tomography and fundus autofluorescence findings complement the phenotypic-genotypic correlations. Other clinical findings, such as hyperopia, enophthalmos, photophobia, sluggish and poorly reactive pupils, the oculodigital sign, keratoconus, and cataract can be the phenotypic manifestations of LCA [5].

\section{Case Presentation}

A 4-year-old full-term girl with an ocular history of LCA presented to the emergency room at our tertiary care facility with a partially dislocated single-piece AcrySof® IQ monofocal intraocular lens (IOL). The next day, the child was examined under sedation and showed a completely dislocated IOL into the anterior chamber (AC) of the left eye (Fig. 1). There was no history of trauma or any other surgical intervention. She had wandering eye movements with the estimated visual acuity (VA) of "light perception" in all quadrants before and after lens dislocation. She underwent lens aspiration and posterior chamber IOL implantation two years ago at another eye care facility.

The patient was initially diagnosed with LCA. Subsequently, genotyping showed a homozygotic variant mutation of the gene GUCY2D. There was no family history of any similar condition. The mother reported a constant eye-poking and repeated rubbing habit of the patient, which is characteristic to LCA patients in an attempt to improve their vision by mechanical retinal stimulation. In the left eye, she had a slight corneal haze, a completely dislocated singlepiece IOL with the capsular bag in the AC with whitish fibrous material behind it, associated with areas of surgically induced iris atrophy. The right eye showed a clear cornea, deep AC with a moderately cataractous lens. Ultrasound B-scan showed a mild optic nerve head elevation with a thickened macular area and no retinal detachment in either eye. The electroretinogram was flat and non-recordable.

IOL had dislocated into the $\mathrm{AC}$ most likely from the constant eye-poking. IOL explantation from the anterior segment was suggested to prevent potential complications, such as uveitisglaucoma-hyphema syndrome, pseudophakic bullous keratopathy, and macular edema. Following a thorough assessment of risks and benefits, IOL explantation was decided (Fig. 2). The 
patient was observed for two postoperative days and discharged with prescriptions of tapering cortisone drops and topical antibiotics. She was provided with aphakic glasses and a follow-up.

\section{Discussion}

GUCY2D was the first gene described in association with LCA. Its mutation commonly causes LCA, accounting for $12 \%$ of all LCA cases [3]. GUCY2D mutation was detected in our case. LCA manifests in the first year of life with sensory nystagmus and severe vision loss, which varies from no light perception to $20 / 50$, depending on the causative genetic mutation [2]. The current understanding of the pathology of LCA is explained by the inability of the eyes to undergo phototransduction, which occurs because of a disruption in the vision cycle. This cycle consists of a series of enzymatic reactions between the retinal pigment epithelium and the neurosensory retina.

Through the cycle, dietary vitamin A is metabolized into 11-cis-retinal, which generates photopigments, known as chromophores. Disturbances in this cycle cause failure of the phototransduction cascade. Each genetic mutation encodes for specific retinal proteins that affect specific pathways in the vision cycle. Disruption in the pathways can lead to retinal degeneration and manifest as LCA. The association between disruption in vitamin A metabolism within the retina and photoreceptor degeneration remains unclear. To date, there is no treatment or cure for LCA. Symptoms can be relieved with correction of the refraction error, and low-vision aids and supportive environment can be provided.

However, gene therapy offers promising results. Currently, there are clinical trials for inherited retinal dystrophies, including LCA. The efficacy and safety of a gene therapy vector made from an adeno-associated virus, administered to the subretinal space, is being studied and evaluated as a possible treatment for LCA due to RPE65 gene mutation in human subjects. RPE65 is a key isomerase and is responsible for converting all-trans-retinyl ester to 11-cisretinol. Without RPE65, 11-cis-retinal levels are reduced, and retinyl esters accumulate in the retinal pigment epithelium, leading to cell dysfunction and death [6]. The data in the present literature for gene therapy demonstrate improved light sensitivity, visual fields, and functional vision under dim lighting conditions in patients with RPE65-mediated inherited retinal dystrophy [7].

A specific behavior known as Franceschetti's oculo-digital sign is a characteristic feature of LCA; this sign consists of repeated pressing, poking, and rubbing of the eyes with knuckles and fingers to stimulate the photoreceptors. This behavior might contribute to deep-set eyes or keratoconus [8]. Our case demonstrated a dislocated IOL into the AC caused by constant and repeated eye-poking in a patient with LCA. A study was conducted in 2015 to review the most common indications for AC explantation. Pseudophakic bullous keratopathy was the most common indication, accounting for $38.6 \%$ of the cases, followed by persistent iritis $(27.8 \%)$, IOL decentration $(13.6 \%)$, glaucoma $(11.4 \%)$, refractive error $(2.3 \%)$, and glare (2.3\%) [9].

Cataract surgery in patients with retinal dystrophy can be challenging because of the uncertainty in vision improvement and possible operative risks. Several studies have demonstrated overall improvement following cataract surgery in patients with RP [10]. However, the outcomes of cataract surgery for LCA remain unknown. A retrospective study was performed on the outcomes and improvement in VA in patients with retinal dystrophies and associated cataract. Steps were taken to avoid potential complications in high-risk 
patients. To minimize photic exposure, microscope light was reduced to $60 \%$, and the light was covered between different steps of the procedure. This study included two eyes with LCA and showed significant improvement in VA after cataract surgery. The most common indications for cataract surgery in patients with retinal dystrophies are decreased central vision affecting the ability to read and increased glare. These patients have a greater incidence of zonular weakness, posterior capsular opacification, and cystoid macular edema, compared with the general population [11]. There is a strong association between RP and lens dislocation, which is presumed to be secondary to zonular weakness and vitreous degeneration.

In our patient, zonular disruption could have occurred because of repeated eye-poking. Because of the low VA in our case, the dislocated IOL was explanted, and the patient was provided with aphakic glasses and a follow-up. Cataract surgery in LCA might be of no to little benefit. If the decision is made to proceed, there are intraoperative preventive measures to minimize the chances of possible future IOL subluxations and dislocations. First, the continuous curvilinear capsulorhexis should be smaller than the optics for a better stability. Machine settings should be adjusted to minimize zonular stress, and the placement of a capsular tension ring can be considered when there is a zonular compromise. Management requires regular follow-up and is case dependent. The conservative approach includes IOL removal and prescribing aphakic glasses as in the case presented here, or proceed with IOL exchange, replacement with an anterior or a sutured posterior chamber IOL, or suturing the IOL through the bag to the iris or the sclera. All of which have their drawbacks and associated possible unnecessary complications given the visual prognosis of our patient.

\title{
Statement of Ethics
}

Consent to publish this case report was not obtained. This report does not contain any personal information that could lead to the identification of the patient. However, an approval from the Institutional Review Board at King Khaled Eye Specialist Hospital was obtained.

\section{Disclosure Statement}

The authors have no conflicts of interest to declare.

\author{
Funding Sources
}

None.

\section{Author Contributions}

All authors attest that they meet the current ICMJE criteria for authorship. 


\section{Case Reports in Ophthalmology}

\begin{tabular}{l|l}
\hline Case Rep Ophthalmol 2020;11:48-53 \\
\hline DOI: 10.1159/000505596 & $\begin{array}{l}\text { @ } 2020 \text { The Author(s). Published by S. Karger AG, Basel } \\
\text { www.karger.com/cop }\end{array}$ \\
\hline
\end{tabular}

Al-Owaid et al: Intraocular Lens Dislocation into the Anterior Chamber because of Repeated Eye-Poking in a Patient with Leber's Congenital Amaurosis

\section{References}

1 Leber T. Ueber Retinitis pigmentosa und angeborene Amaurose. Graefes Arch Clin Exp Ophthalmol. $1869 ; 15(3): 1-25$.

2 Heher KL, Traboulsi EI, Maumenee IH. The natural history of Leber's congenital amaurosis. Age-related findings in 35 patients. Ophthalmology. $1992 \mathrm{Feb}$;99(2):241-5.

3 den Hollander AI, Roepman R, Koenekoop RK, Cremers FP. Leber congenital amaurosis: genes, proteins and disease mechanisms. Prog Retin Eye Res. 2008 Jul;27(4):391-419.

4 Koenekoop RK. An overview of Leber congenital amaurosis: a model to understand human retinal development. Surv Ophthalmol. 2004 Jul-Aug;49(4):379-98.

5 Fazzi E, Signorini SG, Scelsa B, Bova SM, Lanzi G. Leber's congenital amaurosis: an update. Eur J Paediatr Neurol. 2003;7(1):13-22.

6 Hauswirth WW, Aleman TS, Kaushal S, Cideciyan AV, Schwartz SB, Wang L, et al. Treatment of leber congenital amaurosis due to RPE65 mutations by ocular subretinal injection of adeno-associated virus gene vector: short-term results of a phase I trial. Hum Gene Ther. 2008 Oct;19(10):979-90.

7 Russell S, Bennett J, Wellman JA, Chung DC, Yu ZF, Tillman A, et al. Efficacy and safety of voretigene neparvovec (AAV2-hRPE65v2) in patients with RPE65-mediated inherited retinal dystrophy: a randomised, controlled, open-label, phase 3 trial. Lancet. 2017 Aug;390(10097):849-60.

8 De Laey JJ. Leber's congenital amaurosis. Bull Soc Belge Ophtalmol. 1991;241:41-50.

9 Oltulu R, Erşan İ, Şatırtav G, Donbaloglu M, Kerimoğlu H, Özkağnıcı A. Intraocular lens explantation or exchange: indications, postoperative interventions, and outcomes. Arq Bras Oftalmol. 2015 MayJun;78(3):154-7.

10 Dikopf MS, Chow CC, Mieler WF, Tu EY. Cataract extraction outcomes and the prevalence of zonular insufficiency in retinitis pigmentosa. Am J Ophthalmol. 2013 Jul;156(1):82-88.e2.

11 Davies EC, Pineda R 2nd. Cataract surgery outcomes and complications in retinal dystrophy patients. Can J Ophthalmol. 2017 Dec;52(6):543-7.

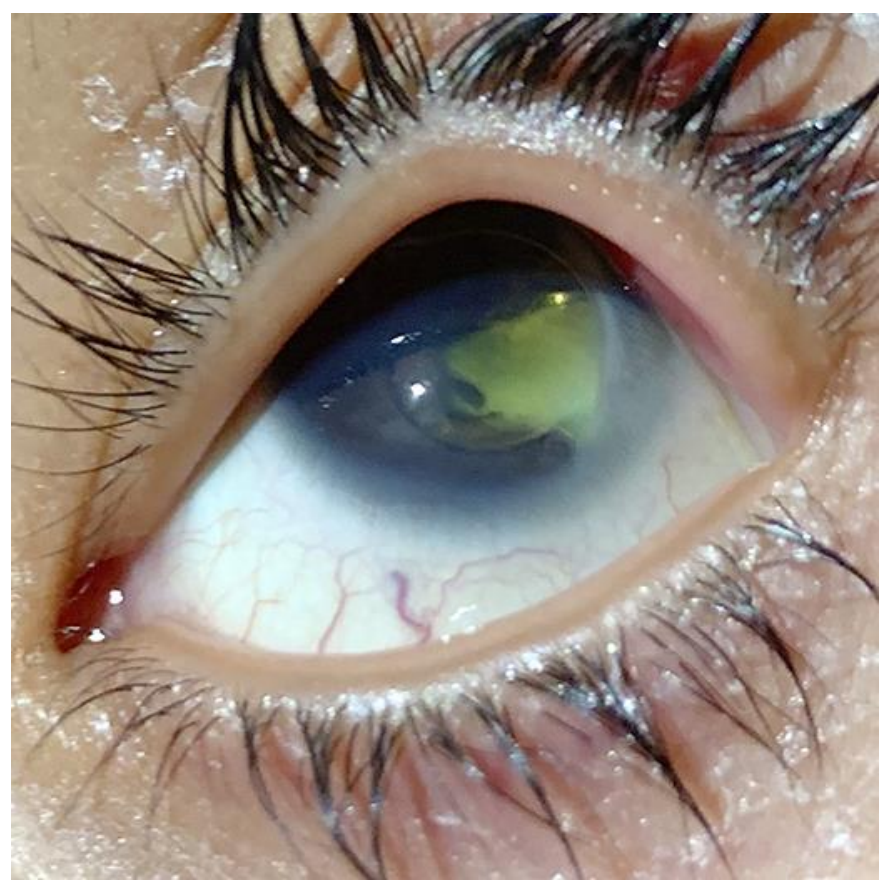

Fig. 1. External photo of the left eye showing a single-piece AcrySof ${ }^{\circledR}$ IQ monofocal intraocular lens dislocated into the anterior chamber, associated with whitish fibrinous material behind and a yellow-green color owing to the blue-filtering properties of the intraocular lens. 


\section{Case Reports in Ophthalmology}
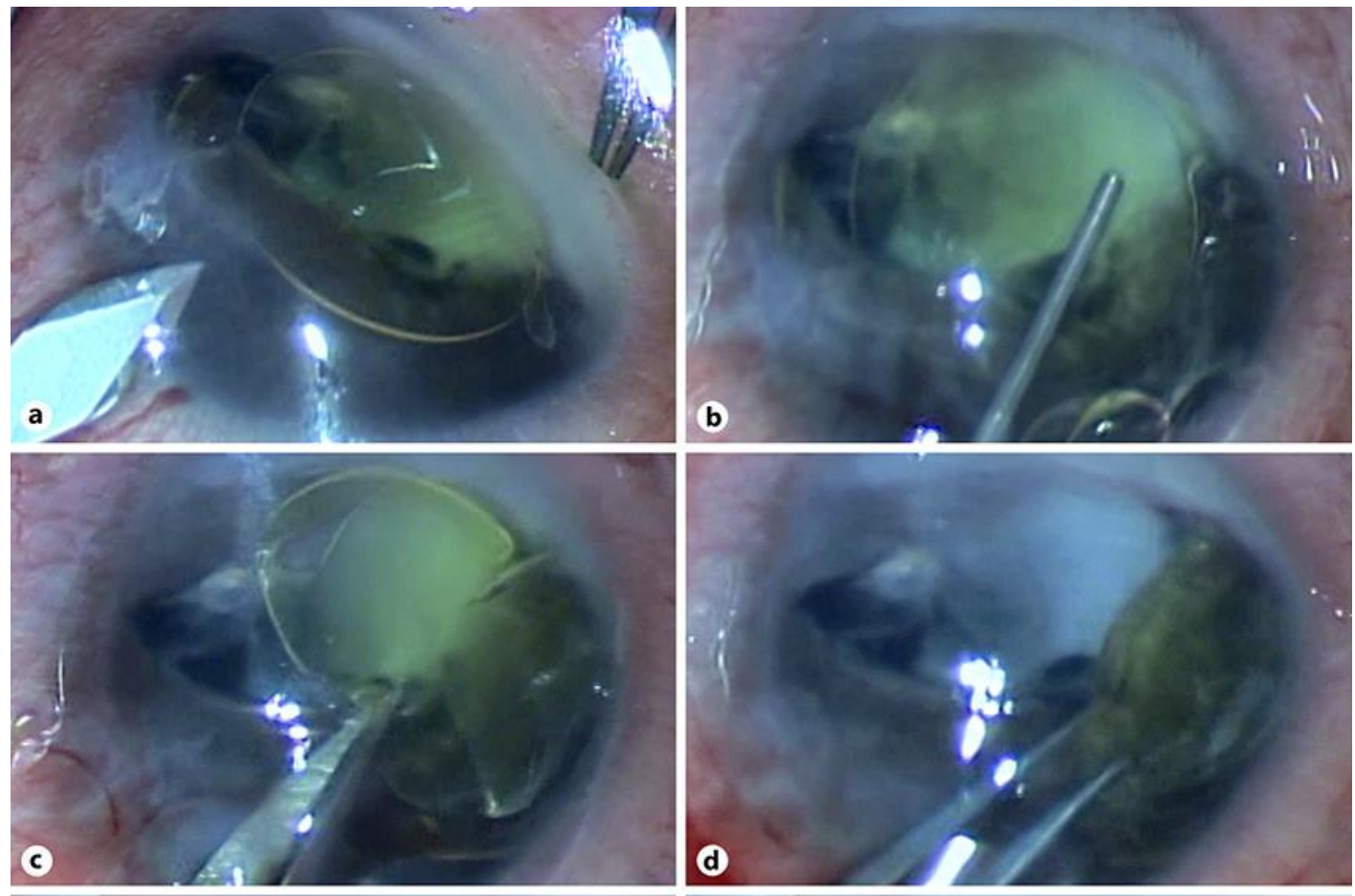

c

Al-Owaid et al: Intraocular Lens Dislocation into the Anterior Chamber because of Repeated Eye-Poking in a Patient with Leber's Congenital Amaurosis
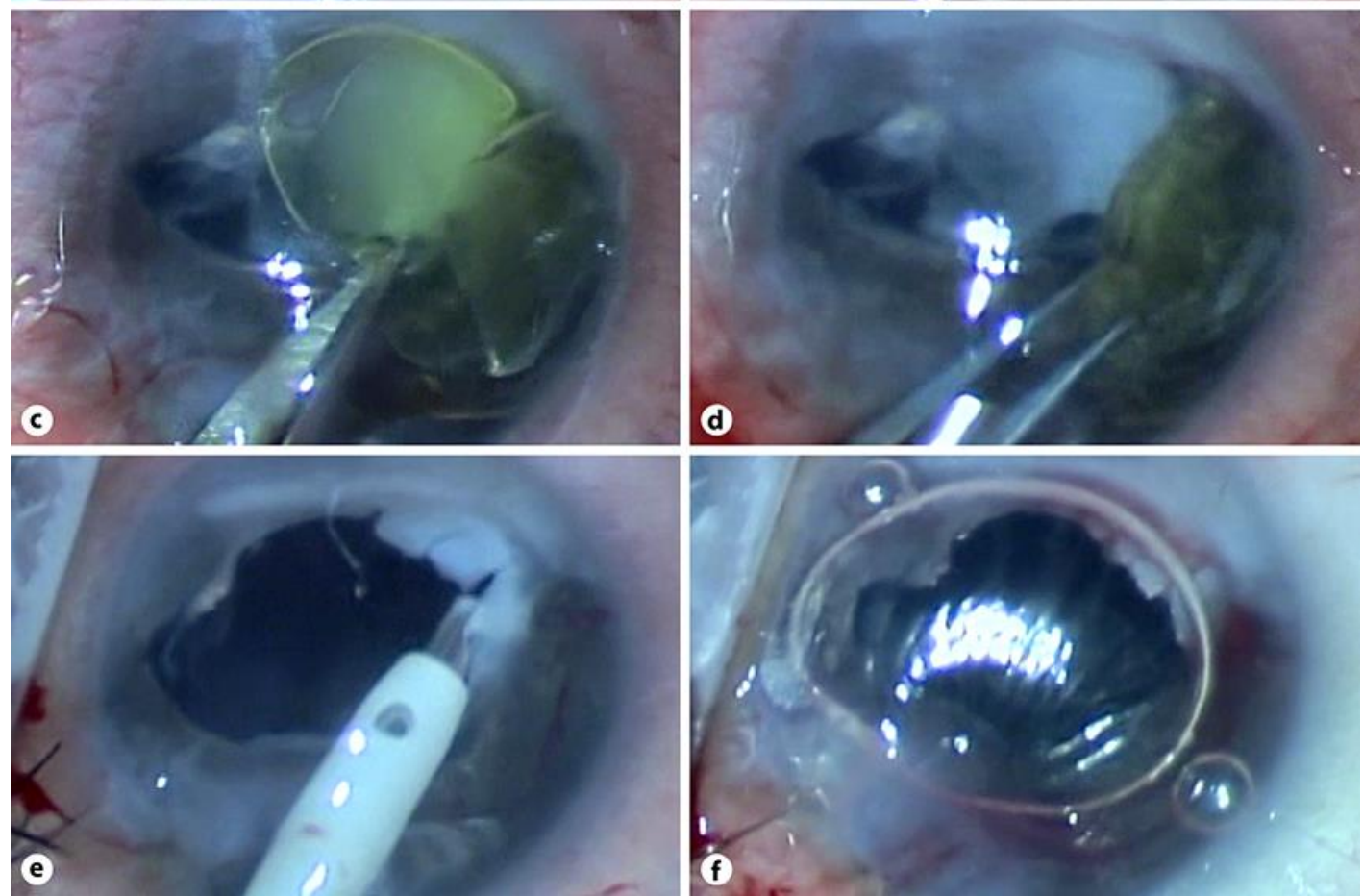

Fig. 2. Surgical photographs of the left eye. a A clear corneal temporal incision was made with a keratome blade. $\mathbf{b}$ Viscoelastic material was inserted to inflate the anterior chamber. c, $\mathbf{d}$ The intraocular lens haptic was moved out of the bag and cut into two halves to facilitate its extraction. e Anterior vitrectomy was performed to remove the membrane covering the pupil. $f$ An air bubble was injected, and the wound was secured with three 10-0 vicryl sutures, followed by subconjunctival cefazolin and dexamethasone injections. 